\title{
Dénoncer la violence. Silences et prise de parole autour du harcèlement sexuel envers les travailleuses domestiques au Brésil
}

Denunciare la violenza. Silenzi e presa di parola attorno alle molestie sessuali verso le lavoratrici domestiche in Brasile

Denouncing violence. Silence and speaking out about sexual harassment against domestic workers in Brazil

\section{Valeria Ribeiro Corossacz}

\section{OpenEdition \\ Journals}

Édition électronique

URL : http://journals.openedition.org/aam/2971

DOI : 10.4000/aam.2971

ISSN : 2038-3215

Éditeur

Dipartimento Culture e Società - Università di Palermo

Référence électronique

Valeria Ribeiro Corossacz, « Dénoncer la violence. Silences et prise de parole autour du harcèlement sexuel envers les travailleuses domestiques au Brésil », Archivio antropologico mediterraneo [En ligne], Anno XXIII, n. 22 (1) | 2020, mis en ligne le 20 juin 2020, consulté le 27 janvier 2021. URL : http:// journals.openedition.org/aam/2971; DOI : https://doi.org/10.4000/aam.2971

Ce document a été généré automatiquement le 27 janvier 2021.

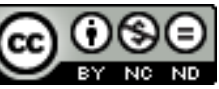

Archivio antropologico mediterraneo è distribuita con Licenza Creative Commons Attribuzione - Non commerciale - Non opere derivate 4.0 Internazionale. 


\section{Dénoncer la violence. Silences et prise de parole autour du harcèlement sexuel envers les travailleuses domestiques au Brésil}

Denunciare la violenza. Silenzi e presa di parola attorno alle molestie sessuali verso le lavoratrici domestiche in Brasile

Denouncing violence. Silence and speaking out about sexual harassment against domestic workers in Brazil

Valeria Ribeiro Corossacz

\section{Objet de la recherche}

1 Cet article présente les données recueillies dans le cadre d'une recherche conduite au Brésil sur les actes de harcèlement sexuel ${ }^{1}$ (assédio sexual) de la part des employeurs sur les travailleuses domestiques, femmes de classe populaire pour la plupart noires. J'examinerai notamment le rôle que joue le silence comme réaction à la violence et dans la définition même du harcèlement sexuel comme objet de recherche.

2 Le harcèlement sexuel contre les travailleuses domestiques est un phénomène répandu dans le monde entier (Nakano Glenn 1992; Vellos 1997; Parreñas 2001; Zarembka 2003 ; Hondagneu-Sotelo 2007 ; Wade 2013 ; Drouilleau 2011)². Au Brésil aussi il existe et même si la plupart des gens, de toutes les classes sociales, le savent, il est difficile d'en évaluer l'ampleur. Comme d'autres aspects des conditions de travail brutales de cette catégorie professionnelle, il s'agit d'une réalité à la fois connue et cachée. Il est donc significatif de faire remarquer que le silence dont je parlerai est un silence partiel, toujours accompagné de moments où on nomme, même rapidement, ce qui doit être tu. Mon intérêt pour la question $\mathrm{du}$ harcèlement sexuel contre les travailleuses domestiques est né d'une précédente enquête parmi un groupe d'hommes blancs de 
classe moyenne de Rio de Janeiro, qui m'avaient parlé de leurs propres actes de harcèlement sexuel, dans leur jeunesse, contre des travailleuses domestiques (Ribeiro Corossacz 2018). J'ai donc décidé d'entendre les travailleuses domestiques. Lorsque j'ai commencé cette recherche, je me suis rapidement aperçue qu'il y avait très peu d'études sur le sujet (DeSouza, Cerqueira 2009; Mori et al. 2011), c'est-à-dire qu'une sorte de silence régnait également dans la production scientifique. Les études sur les travailleuses domestiques se sont développées au cours des trente dernières années, mais cet aspect spécifique n'est jamais abordé que sous forme d'allusion (Vieira 1987; Goldstein 2003 ; Santos 2009 ; Mori et al. 2011). La rareté d'études sur le harcèlement sexuel vécu par les travailleuses domestiques peut s'expliquer par plusieurs éléments. D'abord, la naturalisation de la violence et du harcèlement sexuel envers le femmes noires et pauvres dans le discours dominant sur la valorisation du métissage dans la formation de la société et de l'identité nationales. Dans ce discours, les «relations sexuels » imposées par les maîtres d'origine européenne aux femmes esclaves d'origine africaine sont présentées comme un élément positif ayant permis le caractère métis de la société (Freyre 1933 ; Prado Jr. 1942). Ensuite, la légitimation par la classe moyenne, surtout par les hommes, des actes de harcèlement sexuel (Ribeiro Corossacz 2018), conséquence directe de l'idée que les hommes blancs de classe moyenne ont le droit de faire un usage - y compris sexuel - du corps des travailleuses domestiques (Gonzalez 1983). Enfin, la délégitimation de la parole des travailleuses domestiques, particulièrement forte dans la société brésilienne justement parce qu'il s'agit à la fois de femmes, pauvres, noires et d'origine indigène.

Dans l'histoire du Brésil, colonialité, racisme et sexisme ont toujours été imbriqués de façon structurelle avec l'inégalité de classe typique d'un pays à économie capitaliste (Hasenbalg, Lima et Silva 1999; Guimarães 2002; Lovell 2006; Bruschini, Lombardi 2002). Cette imbrication des rapports de pouvoir est à l'œuvre aussi dans le contexte académique, le principal milieu de production de recherches. Comme l'a fait remarquer Segato (2006) concernant la rareté des études universitaires sur la figure de la babá (nounou), traditionnellement pauvre et noire - et sa remarque vaut pour les études sur la question des travailleuses domestiques - le racisme dans le milieu universitaire a joué un rôle important pour délégitimer, en tant qu'objet de recherche, l'analyse de la violence subie par les femmes pauvres et noires. La présence de ces femmes est fondamentale dans la vie familiale des classes blanches aisées, milieu duquel, avant la mise en place des actions affirmatives dans les universités publiques, provenait la majorité des chercheurs et des chercheuses. Or, tout en cherchant de combler ce silence scientifique sur les expériences de violence vécues par ces femmes dans leur emploi, dans cet article je voudrais analyser également les motifs que peuvent avoir les travailleuses domestiques elles-mêmes pour parler peu de ces violences. Grâce aux luttes des travailleuses domestiques et des femmes noires contre le sexisme, le racisme et leur imbrication, des changements considérables ont eu lieu au cours des dernières décennies, mais il reste difficile d'évaluer l'étendue du phénomène dans le passé comme dans le présent.

\section{Le travail domestique au Brésil}

5 Les travailleuses domestiques sont l'une des catégories les plus vulnérables relativement au droit du travail, pour des raisons historiques - l'héritage de l'esclavage 
- et en raison de la réticence des employeurs actuels à leur reconnaître un statut de travailleuses à part entière et donc à respecter les normes entrées en vigueur aujourd'hui depuis la promulgation de la Loi Complémentaire $n^{\circ} 150$ en en $2015^{3}$. Le salaire mensuel moyen des travailleuses domestiques est inférieur de $60 \%$ au salaire moyen des autres travailleurs. De plus, seules 32 \% d'entre elles sont déclarées (DIEESE 2013). Le Brésil est l'un des pays d'Amérique Latine à avoir mis en place les mesures législatives les plus avancées pour mettre les travailleuses domestiques à égalité avec les autres travailleurs. Toutefois, cette catégorie n'a pas encore les mêmes droits que d'autres catégories professionnelles (OIT/FORLAC 2015). Les améliorations importantes que les travailleuses domestiques ont obtenues sont le résultat de leurs luttes syndicales, de leur ténacité dans la lutte pour la reconnaissance de leurs droits (Bernardino-Costa 2015) et de leur organisation, depuis les années 1930, en associations puis en syndicats.

6 Pour comprendre le travail domestique au Brésil, il faut considérer son lien avec le passé esclavagiste et avec l'origine africaine des esclaves. Après l'abolition légale de l'esclavage en 1888, les esclaves libérées, jusqu'alors confinées à une position de servantes dans les familles de colons blancs ou d'ouvrières agricoles dans de grandes propriétés foncières, ont été insérées dans le marché du travail "libre" dans des positions peu qualifiées. Pour la majorité, elles ont occupé et occupent encore la fonction de travailleuses domestiques. En 2013, plus de cent ans après l'abolition, le Brésil comptait environ 6,2 millions de travailleurs domestiques, parmi lesquels $93 \%$ étaient des femmes et $61 \%$ des femmes noires (DIEESE 2013). Outre les données statistiques, plusieurs chercheuses ont mis en évidence à quel point le travail domestique est associé au fait d'être catégorisée comme noire (Santos-Stubbe 1998 ; Goldstein 2003).

7 La continuité entre la figure de la femme esclave d'origine africaine et la travailleuse domestique est aussi liée au harcèlement sexuel des employeurs et de leurs fils. La littérature est riche de témoignages sur les violences sexuelles perpétrées par les propriétaires d'esclaves et sur l'habitude d'« initier » les fils à la « vie » sexuelle par le viol des femmes mises en esclavage (le texte le plus connu est Maîtres et Esclaves de Freyre, 1933). Cette violence de l'époque de l'esclavage a été présentée comme plus "douce" que dans d'autres pays colonisés et naturalisée par le discours dominant sur le rôle positif du métissage dans la formation de la société et de l'identité nationale, une identité où blancs, noirs et indigènes se "mélangeraient" dans un vide socioéconomique produisant la soi-disant "démocratie raciale". Il existe dans le langage courant plusieurs expressions ${ }^{4}$ et blagues qui renvoient à ce passé de violence, en le présentant toutefois comme un élément folklorique valorisé, effaçant ainsi les rapports de pouvoirs et la souffrance. Les féministes noires ont été les premières à attirer l'attention sur le mécanisme narratif de la formation de l'identité nationale brésilienne: considérant l'histoire non pas du point de vue de l'homme blanc propriétaire terrien, mais du point de vue des femmes mises en esclavage, elles ont dénoncé le fait que les violences qu'elles avaient subies aient été incorporées, en les neutralisant, comme un élément positif et constitutif du discours sur la nation et sur la valorisation du métissage (Gonzalez 1983). Plusieurs travailleuses domestiques que j'ai rencontrées voient une forme de continuité entre les actes de harcèlement sexuel d'aujourd'hui et la violence contre les femmes mises en esclavage d'hier. 
8 Le racisme, le sexisme et l'oppression de classe affectent de façon structurelle la vie des travailleuses domestiques (Oliveira 2008). Une des travailleuses affirme :

Ah, bien sûr que le racisme interfère dans la vie d'une... surtout d'une mère. D'une femme noire, d'une travailleuse domestique. Il interfère de telle façon que la personne commence à se sentir inférieure, incapable de faire certaines choses, comme aller à l'université, se présenter pour un concours dans une institution publique, parce qu'elle est noire et parce qu'elle n'est pas capable de passer un examen.

9 Dans ce propos, le racisme est perçu comme un mécanisme qui fonctionne de manière profonde et structurelle en définissant les secteurs de travail accessibles. Comme le font remarquer De Rudder et Vourc'h : "La domination n'a pas seulement des effets symboliques, comme on le lit trop souvent, elle aliène ceux qui la subissent jusqu'à les faire douter d'eux-mêmes et les contraindre à se conformer au stéréotype qui leur est attribué » $(2006: 192)$.

10 Il est important de rappeler que le racisme opère non seulement en désignant le travail domestique rémunéré comme le seul travail convenant à des femmes pauvres et noires, mais qu'il opère aussi à l'intérieur du travail domestique lui-même : "Alors qu'elles représentent $62 \%$ du total des travailleuses domestiques du pays, les travailleuses domestiques noires percevaient en 2009 une rémunération moyenne de 364,84 R\$, tandis que celle des blanches était en moyenne de 421,58 R\$» (Pinheiro et al. 2011 : 53).

11 Cet « héritage de l'esclavage » combiné à la perception des tâches domestiques comme étant naturellement féminines et à l'idée selon laquelle le travail domestique rétribué implique une disponibilité permanente de la part de la travailleuse (Ávila 2010), ont conduit à la non-reconnaissance des travailleuses domestiques comme travailleuses de plein droit, les excluant du Code du travail adopté en 1943 au motif que leur activité serait un service "non économique et sans but lucratif », avec pour conséquence un refus, pendant plusieurs décennies, de l'État de légiférer dans ce domaine. Face à cette discrimination légale, les travailleuses domestiques se sont organisées pour lutter. Il est fondamental de rappeler que leurs revendications et leurs activités politiques ont été attaquées de plusieurs façons, de la délégitimation à la négation de la reconnaissance de leurs revendications, de l'indifférence à la décrédibilisation, car leurs revendications attentaient et attentent aux inégalités de classe, de race et de sexe dans la société brésilienne. Cette lutte, qui a obtenu beaucoup de victoires législatives, est aujourd'hui encore considérée par de nombreuses travailleuses comme un combat contre une exploitation liée à l'imaginaire esclavagiste.

\section{Le terrain de recherche}

12 Entre 2013 et 2017 j'ai mené une recherche au cours de laquelle j'ai effectué des entretiens avec des activistes du syndicat des travailleurs domestiques de Rio de Janeiro, São Paulo, Campinas, Nova Iguaçu et Natal et avec des travailleuses domestiques de Rio de Janeiro. Les syndicalistes sont elles aussi des travailleuses domestiques, dans la plupart des cas elles travaillent encore ou sont à la retraite, et leur activité syndicale est complètement bénévole. J'ai recueilli les témoignages de 10 syndicalistes, de 20 travailleuses domestiques et de deux avocates qui offrent des services gratuits (dans les locaux du syndicat à Rio de Janeiro et São Paulo). 

50 ans. Les plus âgées ont commencé à travailler vers 8-10 ans, sans avoir fini l'école obligatoire. Sur les vingt travailleuses interrogées, 5 se définissaient comme noires (preta/negra), 6 comme brunes (morena/parda), 5 comme blanches (branca) ou "jaunes" (amarela), avec, parmi ces cinq dernières, une majorité de personnes d'origine nordestin $a^{5}$. Par rapport aux quatre travailleuses auxquelles je n'ai pas eu la possibilité de demander comment elles définissaient leur cor', "couleur", en m'appuyant sur ma classification, je les définirais deux noires, une d'origine nordestina et une blanche. J'ai aussi passé du temps dans le syndicat de Rio à observer les interactions entre syndicalistes et travailleuses et les principaux problèmes discutés lors de leurs rencontres.

Il faut considérer les conditions structurelles dans lesquelles se déroulaient les entretiens : d'un côté des femmes de classe populaire noires ou non-blanches et de l'autre une anthropologue blanche de classe moyenne, brésilienne. En tant que telle, j'étais consciente que je pouvais être identifiée par les femmes rencontrées comme faisant partie du groupe de potentielles employeuses, justement par ma profession de chercheuse et ma "couleur". Au moment de l'entretien, j'ai donc essayé d'être consciente, autant que possible, des implications de ma position. J'ai d'abord essayé de comprendre quand et comment aborder la question du harcèlement. Il a été fondamental pour moi de leur faire comprendre clairement que je condamnais cette violence, car la plupart du temps, comme nous le verrons, les épouses des employeurs harceleurs ne croient pas les travailleuses qui la dénoncent. Je voulais donc que mon interlocutrice sache que je condamnais ce harcèlement, tout en étant consciente que cela n'était pas suffisant pour la mettre à l'aise pour en parler. Ma franchise à poser les questions a peut-être facilité pour elles le choix entre parler ou s'abriter derrière des réponses évasives, ce qui me permettait de comprendre les délimitations et les espaces de dialogue possibles. J'ai ainsi fait face à différents types de réactions : j'ai parfois été surprise de leur capacité à parler de moments très durs, d'autres fois j'ai rencontré une volonté de ne pas en parler que j'ai respectée et d'autres fois encore j'ai partagé des moments de forte émotion et les larmes des femmes harcelées. Il est possible que certaines femmes aient préféré ne pas me parler de ces expériences parce que nous nous connaissions à peine ou bien en raison de ma position de classe, race, et de chercheuse intéressée à connaître leurs conditions de travail et leurs expériences du harcèlement sexuel.

Ces considérations sont importantes non seulement du point de vue ethnographique, mais aussi pour comprendre le contexte de cette recherche, à savoir les possibilités et les obstacles pour accéder aux informations sur le harcèlement sexuel. Si d'un côté il est tout à fait nécessaire d'interroger les femmes à ce sujet, de l'autre il est fondamental de reconnaître qu'il s'agit d'un sujet tabou, dont les gens ne parlent pas, car on pense que la faute repose sur la femme et non sur l'homme.

Cette recherche a donc été empreinte de silence, à plusieurs niveaux, parmi lesquels notamment le silence au cours des entretiens. Comme l'ont remarqué Pinçon et PinçonCharlot « Tout entretien apporte une information, même si elle se réduit au constat du refus de parler » (2002: 40). Ce silence peut se manifester de différentes manières : le silence d'omission, qui ne révèle pas certains événements (dans ce cas la personne ne répond pas), et le silence avec allusion, un silence qui, en fait, évoque certaines situations sans donner des détails et se manifeste aussi de façon non verbale, avec des 
comportements du corps et des expressions du visage qui expriment que la personne interviewée ne souhaite pas continuer à parler. Et il y a aussi le silence de la société, qui nie cette violence ou la ridiculise en la décrivant comme quelque chose qui n'est pas sérieux, pas grave, qui est acceptable. Donc le silence des entretiens peut avoir des significations ambivalentes, voire divergentes. Au long des entretiens, j'ai respecté le silence des femmes, sans faire pression pour les faire parler, parce que leur silence est une réaction à la tendance, tout au long de l'histoire du Brésil, à nier et occulter les diverses formes de violence contre les femmes pauvres et noires.

Bien que le but principal de ma recherche ait justement été de révéler cette forme spécifique de violence vécue par les travailleuses, j'ai fait donc le constat que, dans les cas où je n'étais pas capable de surmonter les barrières sociales rencontrées, j'ai participé moi-même au silence qui entoure la violence.

\section{Obstacles à la dénonciation de la violence}

Dès le début de cette recherche, il est apparu très clairement que la question du harcèlement sexuel était difficile à traiter pour les raisons que je viens de mentionner. Ce type d'agression n'est pas perçu, ni par les syndicalistes ni par les travailleuses, comme le problème principal que pose leur travail, bien que 11 travailleuses sur 20 - et 3 activistes sur 10 - aient déclaré avoir subi du harcèlement sexuel de la part de leur employeur en l'absence de sa femme.

Les syndicalistes pensent que ce phénomène s'est transformé au cours du temps. Leurs propos et ceux des travailleuses permettent de faire l'hypothèse que le phénomène a diminué au cours des dernières années. Parmi les éléments qui peuvent contribuer à ce changement, il faut compter les luttes syndicales pour l'amélioration des conditions de travail, les luttes du mouvement noir contre les discriminations raciales et les politiques d'inclusion sociale des classes les plus désavantagées, auxquelles appartiennent ces travailleuses, promues par les gouvernements du Partido dos Trabalhadores (2003-2016). Ces transformations ont probablement contribué à leur tour à ce que les travailleuses domestiques réagissent plus fortement aux violences, y compris au harcèlement sexuel. Toutefois, en raison de la rareté des données sur cette violence, il est très difficile d'en saisir l'ampleur. Il faut surtout prendre en considération les importantes différences régionales, entre milieu urbain et rural.

À en juger d'après les témoignages recueillis, il semble que le profil de l'homme qui harcèle soit celui d'un homme adulte de classe moyenne - le profil du jeune homme, fils des employeurs est beaucoup moins courant. Il serait nécessaire de recueillir de nouvelles données et d'interroger les jeunes d'aujourd'hui pour vérifier s'il est effectivement moins fréquent que les fils des familles de la classe moyenne blanche harcèlent les travailleuses domestiques et si ces comportements sont encore tolérés ou célébrés comme un modèle positif de masculinité blanche hétérosexuelle des classes moyennes-hautes (Ribeiro Corossacz 2018). Certaines approches savantes relient la légitimation sociale de ces hommes à disposer sexuellement de leurs femmes de ménage, au type de doxa qui a été construite autour de ce partage du milieu domestique, vu comme milieu familier. Il est ainsi possible de penser la travailleuse domestique comme faisant partie de la famille (como se fosse da familia) ${ }^{7}$ et, en même temps, exploiter son travail et son corps considéré comme objet sexuel. Cette réalité d'ailleurs est bien présentée dans la culture populaire médiatisée, dès l'époque des 
films de Pornochanchada ${ }^{8}$ (années 70) jusqu'à l'univers des telenovelas, où on a longtemps laissé sous-entendre l'idée que les travailleuses auraient consenti aux harcèlements sexuels, définis plutôt comme un jeu de séduction. À ce propos, il faut considérer que les femmes rencontrées n'ont pas fait référence à ce type de situation comme un jeu, et qu'elles ne voient pas d'implications amoureuses-érotiques dans une relation qui, pour elles, est de travail.

D'après les syndicalistes, les travailleuses s'adressent rarement au syndicat pour dénoncer les actes de harcèlement sexuel de leurs employeurs. Il s'agit d'un sujet dont il est difficile de parler, même au syndicat, comme le font remarquer les syndicalistes elles-mêmes. Dans un entretien, une syndicaliste de Nova Iguaçu me dit que les travailleuses pensent que parler «ne sert à rien, elles pensent que ça ne vaut pas le coup, parce que c'est leur parole contre la parole de l'employeur et qu'il a davantage d'argent. L'employeuse ne croira pas la travailleuse, elle pensera que c'est elle la responsable, que c'est elle qui a provoqué l'homme. Elles préfèrent quitter leur boulot sans rien dire ». Certaines travailleuses ont aussi souligné ce point : inutile de rapporter la violence vécue, on ne les croira pas. Elenilda, 47 ans, blanche, me raconte son expérience: «Je lui apportais le café et alors il m’a dit : assieds-toi là. Quand je me suis assise sur la chaise, il est arrivé avec son grand truc, là, et moi je me suis échappée en courant. Quand je l'ai dit à ma patronne, elle m'a dit : mais il est médecin, il ne fait pas ce genre de choses ». Le fait que l'employeuse parle de la profession de son mari comme une garantie de son innocence est un exemple des argumentations faisant allusion à la fois à la classe et à la couleur - la classe renvoie toujours à la couleur - en tant que traits sociaux qui, de fait, assurent à certains le privilège de la crédibilité et de l'impunité.

D'après les données recueillies, dans la plupart des cas l'employeuse soit défend son époux, soit le justifie, soit licencie la travailleuse en l'accusant d'être responsable. On peut observer là un renversement typique des responsabilités, selon lequel l'homme n'est pas responsable d'avoir agressé la femme, c'est elle qui l'aurait provoqué ou bien aurait menti. Ce type de renversement, qui n'est pas spécifique au contexte brésilien mais qui est récurrent dans plusieurs situations de viol ou harcèlements vécues par les femmes, permet de produire l'idée qu'au fond, la femme aurait consenti, ou qu'elle aurait pu se dérober, "elle n'aurait pas dû se faire violer » (Mathieu 1991: 149). Puisque dans beaucoup de cas ces femmes ont peur de perdre leur emploi - elles ont besoin du salaire - cette situation produit une véritable limitation de leur liberté d'action et de choix. Une syndicaliste rappelle que le profil des femmes qui ne parlent pas est le plus souvent celui de femmes "seules, célibataires. Elles sont chefs de famille et elles se taisent pour ne pas perdre leur travail, elles ne disent rien ». Donc la difficulté à parler de cette violence s'explique aussi par le fait qu'on ne croit presque jamais ces femmes lorsqu'elles dénoncent les harcèlements.

Dans les entretiens avec les syndicalistes, j'ai pu constater que les sentiments de honte et de peur sont très répandus parmi les travailleuses harcelées. Selon les syndicalistes, la honte et la peur peuvent être un frein qui les empêche de dénoncer ou de parler du harcèlement même avec des proches. Les syndicalistes m'ont dit que dans certains cas elles ont eu l'impression que les femmes avaient été harcelées, mais qu'elles n'arrivaient pas à parler de cette violence lors des rendez-vous au syndicat, même si elles exprimaient beaucoup de rage. Les syndicalistes sont donc en mesure de reconnaître que le fait de ne pas dénoncer et de ne pas révéler la violence peut être une manière de faire face à cette violence et elles sont capables d'accueillir cette façon de 
communiquer. Tout en insistant sur l'importance et la nécessité de dénoncer, les syndicalistes ne culpabilisent pas les femmes qui n'y arrivent pas. Cette attitude est possible parce qu'elles partagent les mêmes conditions de vie que les travailleuses, elles connaissent les oppressions structurelles de classe, sexe et race qui rendraient une action individuelle de dénonciation particulièrement douloureuse et très peu efficace. En outre, éviter de parler d'un événement traumatique peut être lié à la difficulté, insupportable, de revivre l'événement en le racontant, car il s'agit d'un vécu psychologiquement inaccessible (Caruth 1995), mais qui est relativement "accepté » dans la culture dominante, considéré comme pas assez sérieux, qui ne mérite pas d'être verbalisé. Comme le rappellent, entre autres, DeSouza et Cerqueira "être harcelée sexuellement implique un statut inférieur et un manque de pouvoir (powerlessness) » (2009: 1278). Dans un contexte général de négation ou de légitimation des harcèlements sexuels, du racisme, de l'oppression, "le silence semble être vécu comme une forme de désaccord et de résistance », comme le soutient Sheriff au sujet de l'expérience du racisme au Brésil (2000: 125).

La première difficulté touche donc à la possibilité même de révéler le harcèlement sexuel vécu, et d'être crues. Cet élément doit être pris en considération lorsqu'on veut connaître l'ampleur de ce phénomène. Le silence des travailleuses domestiques sur le sujet du harcèlement sexuel doit être corrélé aux obstacles qu'elles rencontrent quand elles nomment ces violences et il ne doit pas être considéré comme un indicateur de son absence ni de leur "consentement".

Cette difficulté à parlers des harcèlements est en fait liée à l'ancrage de la culture de la classe moyenne blanche dans la rhétorique nationale de la valorisation du métissage, qui admet ou tolère les harcèlements sexuels contre les travailleuses domestiques, décrits ou présentés comme un jeu ou comme la manifestation des "besoins sexuels » des jeunes hommes ou d'une sexualité masculine « incontrôlable » (Ribeiro Corossacz 2018). Il faut, de plus, considérer le poids d'une culture, non spécifique du Brésil, qui culpabilise toutes les femmes pour les harcèlements qu'elles subissent, une culture qui peut être reproduite mêmes par les femmes elles-mêmes.

27 Ces premières données aident à comprendre pourquoi les plaintes sont si peu nombreuses. D'après les avocates et les syndicalistes interviewées, rares sont les travailleuses domestiques qui décident de porter plainte pour harcèlement auprès des autorités. Les avocates comme les syndicalistes soulignent comment il est difficile d'intenter ce type de procès : non seulement il est presque impossible de prouver la violence, mais les femmes savent bien qu'en la dénonçant publiquement, elles seront exposées à la stigmatisation qui frappe la femme harcelée ou violée. Après la plainte auprès du syndicat, la plainte aux autorités constitue une étape ultérieure qui risque de n'apporter que frustration et humiliation. Ce choix signifierait rendre publique la violence, subir la stigmatisation qui lui est associée, tout cela pour une probabilité très basse d'obtenir une victoire judiciaire.

28 Les travailleuses et les syndicalistes connaissent donc bien les obstacles et le risque d'exposition à d'autres formes de violence si elles portent plainte ou même simplement si elles parlent de ce qui s'est passé. Elles feraient face à la négation de la violence par l'employeur et l'employeuse, à la banalisation de la violence vécue et à la difficulté d'obtenir justice. Le silence et les allusions, les glissements sur les harcèlements sexuels renvoient ainsi à une condition typique des groupes dominés: «il existe chez les dominés plusieurs types de conscience et de production de connaissance, fragmentés et 
contradictoires, dus justement aux mécanismes mêmes de l'oppression " (Mathieu 1991 : 140-141). Il s'agit donc de reconnaître « les diverses modalités de fragmentation, de contradiction, d'adaptation ou des refus... plus ou moins (dé)structurées de la part des dominé(e)s, modalités dont l'appréhension semble particulièrement malaisée pour un dominant " (Ibidem: 140-141). Le silence devient donc une forme stratégique de réaction, qui révèle la complexité et l'importance des enjeux de ces situations. Les multiples raisons qui amènent à se taire et les différentes peurs qui émergent relèvent du positionnement social multiple de ces femmes, appréhendé grâce au prisme de l'intersectionnalité : racialisées, victimes de violence sexuelle et insérées dans un secteur du marché du travail très informel où les vestiges du passé esclavagiste sont encore présentes et associés aujourd'hui à une exploitation capitaliste (Carneiro 2015; Ávila 2016).

\section{Réactions à la violence}

À en croire les témoignages recueillis, il existe des formes de harcèlement récurrentes : l'homme se présente nu ou avec son sexe en dehors de son pantalon et il essaye d'attraper la travailleuse, laquelle tente de se dérober, parfois en s'enfermant dans une chambre. Il est aussi fréquent que l'homme se frotte sur la femme ou la touche pendant qu'elle travaille (fait la vaisselle ou prépare le repas), ou quand elle achève une tâche qu'il lui a demandé d'accomplir. Dans d'autres cas encore, l'homme entre pendant la nuit dans la chambre où dort la travailleuse et l'agresse.

La plupart des travailleuses que j'ai rencontrées et qui ont été harcelées ont réagi en quittant leur travail sans donner d'explications sur les raisons de leur décision et sans recevoir leur dernier salaire. Ce comportement est également celui qu'a mis en évidence la recherche de Mori (2011). La décision de partir sans dénoncer la violence démontre la profonde conviction chez les travailleuses que parler ne sert à rien. Dans cette situation, les travailleuses optent pour une action libératrice en étant conscientes des difficultés inhérentes aux tentatives de transformer le contexte de travail. Il s'agit d'une forme de révolte contre la violence du harcèlement, mais aussi contre la violence de la délégitimation de leur parole et aussi d'une façon d'exercer leur propre subjectivité. Naira, 50 ans, se définit morena, elle a vécu deux expériences d'agression et elle m'explique qu'en général on cache aux patrons ce qui s'est passé: " on pense toujours que ça nous retombera dessus ». C'est pourquoi quand l'activiste de Nova Iguaçu dit que les travailleuses harcelées "préfèrent quitter leur travail", on comprend que "préférer " est un euphémisme, car elles savent qu'elles n'ont pas d'alternative pour interrompre la violence.

Dans les entretiens, certaines travailleuses ont affirmé que les hommes qui les ont harcelées les menacent pour les empêcher de parler, en leur rappelant que personne ne les croira : c'est la parole de la travailleuse, femme pauvre, noire ou souvent d'origine indigène, contre celle d'un homme de classe moyenne, blanc, considéré comme plus crédible. Révéler le harcèlement sexuel est une forme de réaction, c'est pourquoi les hommes craignent que cela arrive et essaient de l'empêcher. Une travailleuse qui a vécu des expériences de harcèlement sexuel raconte qu'une fois l'homme « avait peur que je parle ». Laura, 54 ans, morena, raconte : «Mais il y en a beaucoup qui se taisent. Moi je lui ai dit : écoute, si tu essaies de me harceler encore une fois, je le dirai à ta femme. Et lui : tu es sûre que tu le feras? Je lui ai répondu : je lui raconterai. Et lui: je te 
virerai... Tu en auras le courage ? Et moi : oui, je l'aurai ». Zezé, 71 ans, originaire du Nordeste, raconte une histoire similaire. Pendant l'une de ses deux agressions, elle demande à son employeur d'arrêter. L'homme lui répond: «Ferme-la !» et elle lui répond : «Je ne me tairai pas, parce que vous êtes là [à vouloir m'agresser] ». Le fait que les hommes ne veuillent pas que les femmes parlent montre qu'ils sont conscients que leur pouvoir n'est pas absolu : les femmes réagissent de différentes façons. Les hommes reconnaissent que l'acte de révéler ces violences représente un geste crucial de rébellion et tentent donc de délégitimer ou de limiter la parole des femmes. Protester, c'est définir ces harcèlements pour ce qu'ils sont, un acte illégitime de violence et d'oppression.

Dans l'entretien avec Dona Zica émerge un autre aspect, central pour comprendre la dynamique du silence autour de ces violences : le rôle que joue la couleur dans le fait de les révéler ou non. Dona Zica est une activiste historique du syndicat de Rio, au moment de l'entretien elle avait 80 ans. D'après elle, les épisodes de harcèlement sexuel ont diminué par rapport au passé, mais elle insiste sur le fait qu'il s'agit d'un problème qui reste «très caché ». Quand elle avait 15 ans elle dormait dans la maison où elle travaillait et, pendant la nuit, le frère de son employeuse essayait d'entrer dans sa chambre. Elle en parle avec sa mère, qui décide de lui faire quitter son emploi, sans donner d'explications aux patrons. Elle me raconte qu' « à cette époque... on se taisait complètement. Ça arrivait et on se taisait ». Puis elle m'explique qu'aujourd'hui ce n'est plus comme ça, le syndicat donne des informations et des indications pour dénoncer. Plus tard au cours de l'entretien elle insiste : «Je n'ai pas protesté. J'ai protesté auprès de ma mère et ma mère m'a fait partir de cette maison ». Quand je lui demande la couleur des personnes qui l'employaient, elle me répond: «Blancs. Une raison de plus de ne pas me croire, non? ». Cette affirmation est la seule, parmi tous les témoignages que j'ai recueillis, qui pose explicitement la question de comment la classification de la couleur d'un individu, dans le sens ample que le terme cor a au Brésil, affecte la définition de qui est crédible pour dénoncer les harcèlements, un problème dont les syndicalistes et les avocates sont conscientes. L'affirmation de Dona Zica sous-entend que les autres membres de la famille ne l'auraient pas crue parce qu'ils étaient blancs et qu'elle était noire. Dona Zica pose ainsi la question de comment la classification de la couleur des personnes impliquées conditionne la possibilité même de révéler la violence. En parler avec sa mère signifie savoir qu'on peut la croire parce qu'elle s'adresse à un réseau de rapports sociaux, à une communauté qui peut reconnaître et comprendre les conditions de travail et d'oppression où elle se trouve. Le long parcours militant de Dona Zica, sa prise de conscience de l'exploitation dans son travail, peuvent expliquer son choix d'évoquer le rôle que joue le racisme pour établir ce qui peut être nommé, ce qui est crédible et ce qui est condamnable.

Dans l'entretien, Dona Zica pose aussi la question de la crédibilité en lien avec le sentiment de culpabilité que de nombreuses travailleuses éprouvent lorsqu'elles vivent des harcèlements : "Dans ces moments, il y a un sentiment de culpabilité de la part de la travailleuse : Pourquoi je n'ai pas lutté ? Pourquoi je n'ai pas parlé ? Pourquoi je n'ai pas fait ceci et cela? En général, le problème est ailleurs : les patrons ne vont pas la croire ». Rompre le silence autour du harcèlement sexuel veut donc dire faire l'expérience de ne pas être crue parce que travailleuse domestique, parce que pauvre, et noire, et tout cela produit l'accusation d'être "menteuse ». La famille qui emploie la travailleuse «va la virer et peut même l'intimider : comment va-t-elle prouver ce qui s'est passé ? Elle est complètement isolée dans son travail. Comment peut-elle 
prouver?». Dona Zica reconnaît la structure objective des rapports de pouvoir dans cette situation et elle peut donc comprendre « l'état d'impuissance objective, matérielle et mentale " (Mathieu $1991: 180$ ) qui fait que les travailleuses n'en parlent pas. Il est donc essentiel de réaffirmer que le sujet opprimé « voulant survivre, il 'refoule' dans l'oppression, ce qui ne veut pas dire qu'il consent à l'oppression » (Ibidem : 187).

\section{Conclusions}

Les données recueillies permettent de comprendre combien il est difficile d'aborder la question des harcèlements sexuels, d'abord pour les travailleuses domestiques ellesmêmes. Cette forme de violence se caractérise par l'imbrication de discriminations structurelles de classe, race et genre et il est souvent trop dur d'y faire face individuellement. Il s'agit d'une violence basée sur le genre qui ne peut pas être isolée d'autres formes de violence, comme l'oppression de classe et le racisme. Derrière les difficultés des travailleuses domestiques à dénoncer les actes de harcèlement sexuel il faut donc reconnaitre le contexte plus large dans lequel elles vivent qui, au niveau individuel, collectif et institutionnel, nie ou délégitime leur vécu d'oppression structurelle et donc leurs mots pour le nommer.

Au cours de ma recherche j'ai pu observer le rôle fondamental joué par le syndicat face à la difficulté à parler du harcèlement sexuel. Les activistes perçoivent la difficulté des travailleuses à le faire comme une forme d'omission parlante, qu'elles respectent parce qu'elles la comprennent, en raison d'une reconnaissance réciproque. Elles sont conscientes de ce qui fait obstacle à la dénonciation et reconnaissent donc le silence comme une « réaction stratégique - plus ou moins efficace - à leur position de relatif manque de pouvoir (powerlessness)" (Gal 1991: 182). C'est exactement ce type de compréhension qui permet de soutenir ces femmes et de créer des alliances, en vue de rompre le silence et de révéler enfin la violence, en reconnaissant que se taire n'est pas consentir. Quand on considère les difficultés des travailleuses à révéler le harcèlement dans le contexte plus large des inégalités de classe, du racisme, du sexisme et des discriminations institutionnelles qu'elles vivent, on comprend que le silence devient une forme de résilience, une façon de dénoncer le niveau d'oppression vécue et le sentiment d'impuissance qui en découle.

\section{BIBLIOGRAPHIE}

Ávila M. B., 2010 « Divisão sexual do trabalho e emprego doméstico no Brasil », in A. Costa et al. (sous la direction de), Divisão sexual do trabalho, estado e crise do capitalismo, SOS Corpo, Recife: 115-144.

Ávila M. B., 2016 « O tempo do trabalho doméstico remunerado: entre cidadania e escravidão », in Abreu A. Rangel de Paiva, Hirata H., Lombardi M. R. (sous la direction de), Gênero e trabalho no Brasil e na França: perspectivas internacionais, Boitempo, São Paulo: 137-149. 
Bernardino-Costa J., 2014 « Intersectionality and female domestic workers' unions in Brazil », in Women's Studies International Forum, XLVI : 72-80.

Brites J., 2007 « Afeto e desigualdade: gênero, geração e classe entre empregadas domésticas e seus empregadores », in Cadernos Pagu, 29: 91-109.

Bruschini C., Lombardi M. R., 2002 « Instruídas e trabalhadeiras. Trabalho feminino no final do século XX », in Cadernos Pagu 17/18: 157-196.

Carneiro S., 2015 « Apresentação », in Bernardino-Costa, J., Saberes Subalternos e Decolonialidade, Editora UnB, Brasilia: 7-11.

Caruth C., 1995 « Introduction », in C. Caruth (ed.), Trauma: explorations in memory, Johns Hopkins University Press, Baltimore: 3-13.

De Rudder V., Vourc'h F., 2006 « Les discriminations racistes dans le monde du travail », in D. Fassin, E. Fassin (sous la direction de), De la question sociale à la question raciale ?, La Découverte, Paris: 183-202.

DIEESE, 2013 « O Emprego Doméstico no Brasil », in Estudos e Pesquisas, 68.

DeSouza E., Cerqueira E., 2009 « From the Kitchen to the Bedroom », in Journal of Interpersonal Violence, XXIV (8): 1264-1284.

Drouilleau F., 2011 Parenté et domesticité féminine à Bogotá, Thèse de doctorat, Anthropologie sociale et historique, EHESS, Paris.

Ehrenreich B., Russel Hochschild A. (eds), 2003 Global Woman: Nannies, Maids and Sex Workers in the New Economy, Metropolitan Books, New York.

Freyre G., 1933 Casa-Grande e Senzala, José Olympio Editor, Rio de Janeiro, trad. fr., Maîtres et Esclaves, Gallimard, Paris, 1974.

Gal S., 1991 « Between Speech and Silence: The Problematics of Research on Language and Gender ", in M. di Leonardo (ed.), Gender at the Crossroads of Knowledge, University of California Press, Berkeley: 175-203.

Goldstein D., 2003 Laughter out of Place. Race, Class, Violence, and Sexuality in a Rio Shantytown, University of California Press, Berkeley.

Gonzalez L., 1983 « Racismo e sexismo na cultura brasileira », in Ciências Sociais Hoje. Anpocs, II: 223-244.

Guimarães A.S., 2002 Classes, Raças e Democracia, Editora 34, São Paulo.

Hasenbalg C., Márcia L., do Valle Silva N., 1999 Cor e Estratificação social, Contra Capa Livraria, Rio de Janeiro.

Hondagneu-Sotelo P., 2007 Doméstica: immigrant workers cleaning and caring in the shadows of affluence, University of California Press, Berkeley.

IPEA, 2011 Situação atual das trabalhadoras domésticas no país, Comunicados do Ipea 90, IPEA, Rio de Janeiro.

Kofes M. S., 2001 Mulher, Mulheres - Identidade, diferença e desigualdade na relação patroas empregadas, Ed. Unicamp, Campinas.

Lovell P. A., 2006 « Race, Gender and Work in São Paulo, Brazil, 1960-2000 », in Latin American Research Review 41: 63-87. 
Mathieu N.-C., 1991 « Quand céder n'est pas consentir. Des déterminants matériels et psychiques de la conscience dominée des femmes, et de quelques-unes de leurs interpértations en ethnologie ", in N.-C. Mathieu, L'anatomie politique. Catégorisations et idéologies du sexe, côté-femmes, Paris: 131-226.

Mori N. et al. (sous la direction de), 2011 Tensões e experiências: Um retrato das trabalhadoras domésticas de Brasília e Salvador, Centro Feminista de Estudos e Assessoria, Brasília.

Motsei M., 1990 The Best Kept Secret: Violence against domestic workers, Paper presented at the Centre for the Study of Violence and Reconciliation, Seminar No. 5, 25 July.

Nakano Glenn E., 1992 « From servitude to service: historical continuities in the racial division of paid reproductive labor », in Signs: Journal of women in culture and society, XVIII : 1-43.

OIT/FORLAC, 2015 Promoción de la formalizatión del trabaljo doméstico en Brasil, Oficina Regional para América Latina y el Caribe.

Oliveira M. C., 2008 « A organização das trabalhadoras domésticas no Brasil », in M. B. Ávila et al. (sous la directions de), Reflexões feministas sobre informalidade e trabalho doméstico, Sos Corpo, Recife :109-116.

Parreñas Salazar R., 2001 Servants of globalization: women, migration and domestic work, Stanford University Press, Stanford.

Pinçon M., Pinçon-Charlot M., 2002 Voyage en grande bourgeoisie, PUF, Paris.

Pinheiro L., et al., 2011 « Situação atual das trabalhadoras domésticas no país », in N. Mori, S. et al. (sous la direction de), Tensões e experiências: Um retrato das trabalhadoras domésticas de Brasilia e Salvador, Centro Feminista de Estudos e Assessoria, Brasília : 33-69.

Prado Junior C., 1942 Formação do Brasil contemporâneo, Editora Brasiliense, Rio de Janeiro.

Ribeiro Corossacz V., 2018 White middle-class men in Rio de Janeiro. The making of a dominant subject, Lexington Books, Lanham.

Ribeiro Corossacz V., 2020 « "Domestic work is work". Le lotte delle lavoratrici domestiche e la divisione socio-sessuata del lavoro", in B. Busi (sous la directions de), Separate in casa. Femministe, sindacaliste e lavoratrici domestiche a confronto, Ediesse, Roma: 85-103.

Sansone L., 2003 Blackness without Ethnicity. Constructing Race in Brazil, Palgrave Macmillian, New York.

Santos R. de Jesus dos, 2009 Corpos domesticados: a violência de gênero no cotidiano das domésticas em Montes Claros - 1959 a 1983, Dissertação (Mestrado em História Social), Programa de Pós-Graduação em História Social, Universidade Federal de Uberlândia, Uberlândia.

Santos-Stubbe C. dos, 1998 « Cor, cultura e sociedade: a questão da etnicidade entre as empregadas domésticas ", in Estudos Afro-Asiáticos, XXXIII : 51-69.

Segato R. L., 2006 « O Édipo brasileiro: a dupla negação de gênero e raça », in Série Antropologia, CD: $1-21$.

Sheriff R., 2000 « Exposing Silence as Cultural Censorship: a Brazilian Case », in American Anthropologist, CII (1) : 114-132.

Vellos D., 1997 « Immigrant Latina Domestic Workers and Sexual Harassment », in Journal of Gender \& The Law, V (2) : 407-432.

Vieira Celma R., 1987 « Negra: mulher e doméstica - considerações sobre as relações sociais no emprego doméstico », in Estudos Afro-Asiáticos, XIV : 141-158. 
Wade P., 2013 « Articulation of erotization and race: Domestic Service in Latin America ", in Feminist Theory, XIV (2) : 187-202.

Zarembka J., 2003 « America's Dirty Work: Migrants Maids and Modern-Day Slavery », in B. Ehrenreich, A. Russel Hochschild (eds), Global Woman: Nannies, Maids and Sex Workers in the New Economy, Metropolitan Books, New York : 142-153.

\section{NOTES}

1. J'ai traduit assédio sexual, expression employée couramment par les femmes, par harcèlement sexuel. Dans les entretiens, les femmes et moi même n'avons pas cherché une définition juridique. J'ai considéré comme harcèlement sexuel tout comportement et acte physique et verbal de caractère sexuel non souhaité accompli par un homme de la famille employeuse. Dans certaines conversations, je n'ai pas employé assédio sexual, mais j'ai choisi d'employer l'expression faltar de respeito (manquer de respect) pour aborder le sujet, en étant consciente de la difficulté d'en parler ouvertement.

2. Pour une analyse du travail domestique rémunéré et non, voir aussi Ribeiro Corossacz 2020.

3. Cette loi garantit un certain nombre de droits du travail, parmi lesquels l'obligation pour l'employeur de signer un contrat de travail dans les premières 48 heures, le salaire minimum, la limitation de la journée de travail à 8 heures et 44 heures hebdomadaires, la compensation salariale pour le travail de nuit et les heures supplémentaires, l'accès à la retraite et au chômage.

4. Par exemple l'expression ter um pé na cozinha (avoir un pied dans la cuisine), utilisée pour parler de personnes considérées comme blanches qui ont un lien de parenté avec la femme mise en esclavage ou la travailleuse domestique noire.

5. Terme employé pour parler des personnes venant du Nordeste, la région la plus pauvre du pays, où prévalent les origines indigènes, qui peuvent avoir des teintes de peau claire. Les nordestinos occupent une place subalterne dans l'histoire des migrations de travail.

6. Au Brésil la classification raciale d'un individu est déterminée par plusieurs éléments : la texture des cheveux, la forme du nez, la couleur de la peau, et la classe sociale. Il faut noter que la plupart de gens emploie dans le langage quotidien le terme "couleur » (cor) pour se référer à tous ces éléments (à ce sujet la littérature est très riche, voir par exemple Sansone 2003 et Guimarães 2002).

7. Pour une analyse de la coexistence d'affect et d'inégalité dans le travail domestique voir Brites 2007 et Kofes 2001, mais surtout les analyses des syndicalistes qui ne sont pas publiées. L'expression "comme étant partie de la famille" est récurrente dans plusieurs autres pays, voir, parmi les plusieurs références, Parreñas 2001.

8. Il s'agit d'une production cinématographique des années 1970 qui mélange le style de la comédie faite de humour naif et burlesque avec des éléments quasi pornographiques. Plusieurs films de ce genre présentent, par un style comique, ce qui est mis en scène comme un simple jeu inoffensif de « séduction » de la parte de l'employeur envers la travailleuse domestique. 


\section{RÉSUMÉS}

L'article présente une recherche qui a été conduite au Brésil parmi des travailleuses domestiques et des syndicalistes sur le harcèlement sexuel de la part des employeurs. Les données recueillies indiquent la difficulté, pour différentes raisons, à toucher ce sujet, à la fois dans la littérature scientifique, dans les relations entre syndicalistes et travailleuses, et pendant l'entretien. Le silence est une des façons de réagir aux multiples formes de violence qui sont présentes dans le harcèlement sexuel, une réponse stratégique qui s'enracine dans les conditions de vie des travailleuses. L'article montre, en fait, que les expériences de harcèlement sexuel ne peuvent être comprises que si on élargit l'analyse aux conditions structurelles de vie des travailleuses domestiques, marquées par le racisme, l'inégalité de classe, et le sexisme, et que celles-ci déterminent en même temps les réactions qui sont mises en place par ces femmes.

L'articolo presenta una ricerca condotta in Brasile tra lavoratrici domestiche e sindacaliste sulle molestie sessuali da parte dei datori di lavoro. Dai dati raccolti emerge la difficoltà, per ragioni diverse, a trattare questo argomento, sia nella letteratura scientifica, sia nelle relazioni tra sindacaliste e lavoratrici, sia durante l'intervista. Il silenzio è uno dei modi di reagire alle molteplici forme di violenza presenti nelle molestie sessuali, una risposta strategica radicata nelle condizioni di vita delle lavoratrici. L'articolo dimostra, infatti, che le esperienze di molestie sessuali possono essere comprese solo se ricondotte alle condizioni strutturali di vita delle lavoratrici domestiche, segnate dal razzismo, diseguaglianza di classe e sessismo, che a loro volta determinano le reazioni messe in campo da esse.

In this paper I present findings of a research carried out in Brazil with domestic workers and union activists concerning the problem of sexual harassment against domestic workers by the male employers. Findings demonstrate how difficult it is to address this problem, for different reasons, in academic literature, in the relationship between domestic workers and union activists, and in the interviews. Domestic workers silence is a way to react to the multiple forms of violence experienced in sexual harassment, a strategic response based on their life conditions. The text discusses in fact how the imbrication of racism, sexism and class inequalities is a key element to understand this sexual harassment, as well women's responses to it.

\section{INDEX}

Keywords : Brazil, domestic workers, racism, sexual harassment, silence

Mots-clés : Brésil, travailleuses domestiques, racisme, harcèlement sexuel, silence

Parole chiave : Brasile, lavoratrici domestiche, razzismo, molestie sessuali, silenzio

\section{AUTEUR}

\section{VALERIA RIBEIRO COROSSACZ}

Dipartimento di Studi Linguistici e Culturali, Università degli Studi di Modena e Reggio Emilia valeria.ribeirocorossacz@unimore.it 